\title{
Four-body adiabatic model applied to elastic scattering
}

\author{
J.A. Christley, J.S. Al-Khalili, J.A. Tostevin and R.C. Johnson \\ Department of Physics, University of Surrey, Guildford GU2 5XH, UK
}

\begin{abstract}
Elastic scattering of a projectile, composed of three clusters, from a spherical target is analysed using a four-body adiabatic model. The model is a generalisation of the three-body adiabatic model of Johnson and Soper. Calculations of the elastic scattering of ${ }^{11} \mathrm{Li}$ from ${ }^{12} \mathrm{C}$ are compared to both experimental data and the results of recent four-body eikonal model calculations.
\end{abstract}

PACS numbers: $\quad$ 24.10.-i, 24.10.Eq, 24.50.+g, 25.70.Bc

Keyword list: $\quad$ Adiabatic, Coupled Channels, elastic scattering, 11Li.

Corresponding Author: Dr. James Alan Christley

Department of Physics

University of Surrey

Guildford, Surrey

GU2 5XH, UK

email: $\quad$ phs1jc@ph.surrey.ac.uk

phone: $\quad$ (+44) 1483300800 ext 2714

fax: $\quad(+44) 1483259501$ 
submitted:

revised:
5 February 1997

14 March 1997 


\section{INTRODUCTION}

Recent four-body scattering model calculations of the elastic scattering of ${ }^{11} \mathrm{Li}$ from a ${ }^{12} \mathrm{C}$ target at $57.9 \mathrm{MeV} / \mathrm{A}$ have shown large effects due to the coupling to projectile breakup channels [1]. The four-body model treats the projectile as a three-body structure $\left({ }^{9} \mathrm{Li}+\mathrm{n}+\mathrm{n}\right)$, and can include the effects of neutron pairing correlations. The calculations of ref. [1] were performed using a four-body eikonal scattering model, which makes high energy and small angle approximations. There is interest in studying the quantitative accuracy of these approximations, and also in studying reactions of weakly-bound systems at lower energies, where transfer reactions and other channel coupling effects have been shown to be important for stable nuclei [2]. It is therefore essential to develop scattering models which are suitable for lower energies, and which are able to assess the accuracy of more approximate and efficient methods.

In this paper we extend the adiabatic model first described by Johnson and Soper [3] to describe reactions of two-body projectiles. It is assumed that the internal motion of the $\mathrm{N}$-clusters within the projectile is slow compared to the relative motion of the projectile and target. We can then freeze the internal coordinates for the duration of the interaction. With this approximation the $(\mathrm{N}+1)$-body wave equation reduces to a set of two-body coupled channels equations, with parametric dependence on the (N-1) internal coordinate vectors, $\vec{x}$. Observables are obtained by evaluating matrix elements of calculated internal coordinate dependent amplitudes between initial and final states:

$$
\left(\frac{\mathrm{d} \sigma}{\mathrm{d} \Omega}\right)_{i_{\mathrm{o}}}=\left(\frac{v_{i}}{v_{\mathrm{o}}}\right)\left|f_{i_{\mathrm{o}}}\right|^{2}
$$

where

$$
f_{i \mathrm{o}}=\int \mathrm{d} \vec{x}_{1} \ldots \mathrm{d} \vec{x}_{N-1} \Phi_{i}^{*}\left(\vec{x}_{1}, \ldots, \vec{x}_{N-1}\right) f_{i \circ}\left(\vec{x}_{1}, \ldots, \vec{x}_{N-1}\right) \Phi_{\mathrm{o}}\left(\vec{x}_{1}, \ldots, \vec{x}_{N-1}\right)
$$

The adiabatic model is equivalent to including coupling to a complete set of excited states, including breakup states, but assuming all states are degenerate. It has been successfully applied to scattering of projectiles composed of two clusters (e.g. deuteron [4], ${ }^{6} \mathrm{Li}[5],{ }^{7} \mathrm{Li}$ [6]). In this paper we extend and apply the model for the scattering of projectiles composed of three clusters. 
The Coupled Discretized Continuum Channels (CDCC) method [7] has superseded the three-body adiabatic model for many low energy reaction studies. However this method does not generalise easily for the three cluster projectile case considered here. The method presented here is a possible way forward which correctly includes many of the features of the few-body dynamics.

We will outline the essential theoretical elements of our implementation, and apply it to the elastic scattering of ${ }^{11} \mathrm{Li}$ from a ${ }^{12} \mathrm{C}$ target, which has recently been studied using a four-body eikonal model [1]. We will use the same physical inputs as the eikonal study in order to compare the two scattering models. The calculated elastic cross sections will be compared to quasi-elastic experimental data from ref. [8]. From the structure point of view ${ }^{11} \mathrm{Li}$ is of interest because it has an extended two-neutron halo, and its three-body structure has been investigated extensively (e.g. [9]).

The eikonal model of ref. [1] is based on two approximations: the semi-classical eikonal approximation, which assumes that the individual clusters in the projectile follow a straight-line trajectory through the interaction region; and the adiabatic approximation, which freezes the internal coordinates. In the eikonal model the composite projectile scattering amplitude is obtained by averaging the eikonal approximations to the cluster target S-matrices over the projectile density for each projectile impact parameter. This method implicitly includes coupling to all breakup angular momenta between the projectile clusters.

The four-body model presented here does not require the eikonal approximation, and solves the scattering problem as a set of coupled channels radial equations in which each breakup relative angular momentum appears as a separate channel. This allows the individual contributions of coupling to each breakup angular momentum to be investigated, and also provides a scattering wavefunction (which is not calculated in the eikonal approach). The wavefunction can be used to calculate transfer cross sections, or as the basis for quasiadiabatic corrections (in the spirit of [10]). The price paid is that the coupled channels calculations are very computer intensive, particularly at high energies where high angular momentum couplings may be important.

The eikonal method is essentially a high energy, small angle approximation, and the adiabatic approximation is usually thought of as a high energy approximation. Experience 
with deuteron scattering [4] suggests that the adiabatic model is accurate for projectile incident energies greater than 25-30 MeV/A, which is of the same order as the estimated lower energy limit for the eikonal model [1]. Further work is in progress to compare the adiabatic model with calculations in which non-eikonal corrections are added systematically to the analysis of [1]. Such a comparison has recently proved very instructive for two-body projectiles [11]. The adiabatic method is 'exact' in the sense that it is purely quantum mechanical. It may not prove to be as efficient as the eikonal-based methods, but it will provide an important benchmark with which to assess more approximate techniques.

\section{DETAILS OF THE FOUR-BODY ADIABATIC MODEL}

\section{A. The four-body wave equation}

The adiabatic models for two and three cluster projectiles share the same theoretical basis. In the former we freeze the relative coordinate between two clusters and in the latter we freeze the two Jacobi coordinates that describe the three-body system (labelled $\vec{\rho}$ and $\vec{r}$ in figure 1). In our coordinate system $\vec{R}$ is the relative coordinate between the target (T) and the centre of mass of the projectile.

The exact four-body wave equation for scattering of a three cluster projectile from a inert spherical target can be written

$$
\left[E-h(\vec{\rho}, \vec{r})-T_{R}-V(\vec{R}, \vec{\rho}, \vec{r})\right] \Psi(\vec{R}, \vec{\rho}, \vec{r})=0
$$

where $h(\vec{\rho}, \vec{r})$ is the internal hamiltonian of the projectile, and the potential $V(\vec{R}, \vec{\rho}, \vec{r})$ is the sum of the two-body potentials between the target and each cluster in the projectile. We make a coupled channels expansion of the wavefunction in terms of the ground state o and inelastic and breakup states $i$ of the projectile (which together form a complete set):

$$
\Psi(\vec{R}, \vec{\rho}, \vec{r})=\Phi_{\mathrm{o}}(\vec{\rho}, \vec{r}) \chi_{\mathrm{o}}(\vec{R})+\sum_{i} \Phi_{i}(\vec{\rho}, \vec{r}) \chi_{i}(\vec{R})
$$

where $\Phi$ are orthonormal eigenstates of the internal hamiltonian

$$
h(\vec{\rho}, \vec{r}) \Phi_{j}(\vec{\rho}, \vec{r})=\epsilon_{j} \Phi_{j}(\vec{\rho}, \vec{r}) \quad ; \quad\left\langle\Phi_{j}(\vec{\rho}, \vec{r}) \mid \Phi_{i}(\vec{\rho}, \vec{r})\right\rangle=\delta_{i j}
$$


Substituting eq. 4 into the wave equation (eq. 3) yields

$$
\begin{aligned}
& {\left[E-\epsilon_{\mathrm{o}}-T_{R}-V(\vec{R}, \vec{\rho}, \vec{r})\right] \Phi_{\mathrm{o}}(\vec{\rho}, \vec{r}) \chi_{\mathrm{o}}(\vec{R})+} \\
& \sum_{i}\left[E-\epsilon_{i}-T_{R}-V(\vec{R}, \vec{\rho}, \vec{r})\right] \Phi_{i}(\vec{\rho}, \vec{r}) \chi_{i}(\vec{R})=0 .
\end{aligned}
$$

If at this point we introduce the adiabatic approximation, and replace $\epsilon_{i}$ by the ground state energy $\epsilon_{\mathrm{o}}$, then eq. 6 has the structure:

$$
\left(E-\epsilon_{\mathrm{o}}-T_{R}\right) \Psi^{\mathrm{adia}}(\vec{R}, \vec{\rho}, \vec{r})=V(\vec{R}, \vec{\rho}, \vec{r}) \Psi^{\mathrm{adia}}(\vec{R}, \vec{\rho}, \vec{r})
$$

where the adiabatic wavefunction $\Psi^{\text {adia }}$ is a superposition of the ground state and all excited states of the projectile

$$
\Psi^{\mathrm{adia}}(\vec{R}, \vec{\rho}, \vec{r})=\Phi_{\mathrm{o}}(\vec{\rho}, \vec{r}) \chi_{\mathrm{o}}^{\mathrm{adia}}(\vec{R})+\sum_{i} \Phi_{i}(\vec{\rho}, \vec{r}) \chi_{i}^{\mathrm{adia}}(\vec{R})
$$

This wavefunction has the asymptotic form of a pure Coulomb distorted wave $\Psi_{\vec{K}_{\mathrm{o}}}^{\text {Coul }}$, with the projectile in the ground state, in the elastic channel, plus outgoing waves in all channels

$$
\Psi^{\text {adia }}(\vec{R}, \vec{\rho}, \vec{r}) \stackrel{R \rightarrow \infty}{\longrightarrow} \Phi_{\mathrm{o}}(\vec{\rho}, \vec{r}) \Psi_{\vec{K}_{\mathrm{o}}}^{\mathrm{Coul}}(\vec{R})+\text { (outgoing waves) }
$$

The adiabatic wave equation (7) has only a parametric dependence on the internal coordinates and can be solved as a two-body dynamical problem for fixed $\rho$ and $r$. In the next sections we describe the partial wave expansion of the wavefunction and potentials before describing how the observables are calculated. In the remainder of this paper we drop the now implied superscript 'adia', and assume $\epsilon \equiv \epsilon_{\mathrm{o}}$ and $\vec{K} \equiv \vec{K}_{\mathrm{o}}$.

\section{B. Partial wave expansion}

The coupling scheme we adopt for the partial wave expansion of the wavefunction is $\left(l_{1}, l_{2}\right) \mathcal{L}, L ; J$ where: $l_{1}$ is the relative angular momentum between the two valence neutrons (1) and $(2) ; l_{2}$ is the relative angular momentum between the valence neutrons and the core (C); and $L$ is the relative angular momentum of the projectile and target $(\mathrm{T})$ (see figure $1)$. Using the shorthand notation for channel $\alpha \equiv\left(l_{1}, l_{2}\right) \mathcal{L}, L$ we can expand the adiabatic wavefunction

$$
\Psi(\vec{R}, \vec{\rho}, \vec{r})=\sum_{\alpha \alpha^{\prime} J M_{J}} \mathcal{C}_{\alpha}^{J M_{J}}(\rho, r) \frac{\mathcal{X}_{\alpha^{\prime}: \alpha}^{J}(R, \rho, r)}{K R}\left[\left[Y_{l_{1}^{\prime}}(\hat{r}) \otimes Y_{l_{2}^{\prime}}(\hat{\rho})\right]_{\mathcal{L}^{\prime}} \otimes Y_{L^{\prime}}(\hat{R})\right]_{J M_{J}}
$$


where the coefficients $\mathcal{C}$ must be chosen to give the correct asymptotic boundary conditions (eq. 9) with a pure Coulomb distorted wave, with the projectile in the ground state, in the elastic channel, and outgoing waves in all channels. These coefficients depend on the ground state wavefunction and will be evaluated in section 2.4 .

The radial functions $\mathcal{X}$ are solutions of the coupled radial equations (for fixed $\rho$ and $r$ )

$$
\begin{array}{r}
{\left[\left(E-\epsilon_{\mathrm{o}}\right)+\frac{\hbar^{2}}{2 \mu}\left(\frac{\mathrm{d}^{2}}{\mathrm{~d} R^{2}}-\frac{L^{\prime}\left(L^{\prime}+1\right)}{R^{2}}\right)-V_{C}(R)\right] \mathcal{X}_{\alpha^{\prime}: \alpha}^{J}(R, \rho, r)=} \\
\sum_{\alpha^{\prime \prime}}\left\langle\alpha^{\prime} ; J|V(\vec{R}, \vec{\rho}, \vec{r})| \alpha^{\prime \prime} ; J\right\rangle \mathcal{X}_{\alpha^{\prime \prime}: \alpha}^{J}(R, \rho, r),
\end{array}
$$

subject to the asymptotic boundary conditions

$$
\mathcal{X}_{\alpha^{\prime}: \alpha}^{J}(R, \rho, r) \stackrel{R \rightarrow \infty}{\longrightarrow} \delta_{\alpha^{\prime} \alpha} F_{L}(K R)+M_{\alpha^{\prime}: \alpha}^{J}(\rho, r) H_{L^{\prime}}^{+}(K R)
$$

Note that the channels $\alpha^{\prime}$ in our expansion of the wavefunction (10) are defined purely by the relative angular momenta of the clusters and the target. The channels do not correspond to particular excited states of the projectile at particular excitation energies, but instead to a degenerate representation of the complete set of states for that configuration. An advantage of the adiabatic approach is that we do not require knowledge of the excited states of the projectile.

\section{The four-body potential matrix elements}

The potential matrix elements entering eq. (11) are

$$
\begin{array}{r}
\left\langle\alpha^{\prime} ; J|V(\vec{R}, \vec{\rho}, \vec{r})| \alpha^{\prime \prime} ; J\right\rangle=\iiint \mathrm{d} \hat{R} \mathrm{~d} \hat{\rho} \mathrm{d} \hat{r}\left[\left[Y_{l_{1}^{\prime}}(\hat{r}) \otimes Y_{l_{2}^{\prime}}(\hat{\rho})\right]_{\mathcal{L}^{\prime}} \otimes Y_{L^{\prime}}(\hat{R})\right]_{J M_{J}}^{*} \\
V(\vec{R}, \vec{\rho}, \vec{r})\left[\left[Y_{l_{1}^{\prime \prime}}(\hat{r}) \otimes Y_{l_{2}^{\prime \prime}}(\hat{\rho})\right]_{\mathcal{L}^{\prime \prime}} \otimes Y_{L^{\prime \prime}}(\hat{R})\right]_{J M_{J}}
\end{array}
$$

We assume central potentials and ignore any spin dependence. The coupling potential, the sum of the potentials between the target $(\mathrm{T})$ and the core $(\mathrm{C})$ and two valence particles $(1)$ and (2) of the projectile, is:

$$
\begin{aligned}
& V(\vec{R}, \vec{\rho}, \vec{r})=V_{\mathrm{CT}}\left(\left|\vec{R}-\frac{m_{1}+m_{2}}{m_{P}} \vec{\rho}\right|\right) \\
& \quad+V_{1 \mathrm{~T}}\left(\left|\vec{R}+\frac{m_{C}}{m_{P}} \vec{\rho}+\frac{m_{2}}{m_{1}+m_{2}} \vec{r}\right|\right)+V_{2 \mathrm{~T}}\left(\left|\vec{R}+\frac{m_{C}}{m_{P}} \vec{\rho}-\frac{m_{1}}{m_{1}+m_{2}} \vec{r}\right|\right) .
\end{aligned}
$$


For clarity we suppress the mass ratios from the further expressions. The first term $V_{\mathrm{CT}}$, a function of two vectors $\vec{R}$ and $\vec{\rho}$, can be expressed as a multipole expansion

$$
V_{\mathrm{CT}}(|\vec{R}+\vec{\rho}|)=\sum_{k} V_{k}^{\mathrm{CT}}(R, \rho)\left[Y_{k}(\hat{R}) \otimes Y_{k}(\hat{\rho})\right]_{00}
$$

with matrix elements

$$
\begin{aligned}
\left\langle\alpha^{\prime} ; J\left|V_{\mathrm{CT}}(\vec{R}, \vec{\rho})\right| \alpha^{\prime \prime}: J\right\rangle=\delta_{l_{1}^{\prime} l_{1}^{\prime \prime}} \sum_{k} \frac{V_{k}^{\mathrm{CT}}(R, \rho)}{(2 k+1)} \\
W\left(\mathcal{L}^{\prime \prime} L^{\prime \prime} \mathcal{L}^{\prime} L^{\prime} ; J k\right)\left\langle L^{\prime \prime} 0 L^{\prime} 0 \mid k 0\right\rangle\left\langle l_{2}^{\prime \prime} 0 l_{2}^{\prime} 0 \mid k 0\right\rangle \\
(-)^{J-\mathcal{L}^{\prime \prime}-l_{2}^{\prime}-k_{R}} \hat{L}^{\prime} \hat{L}^{\prime \prime} \hat{l}_{2}^{\prime} \hat{l}_{2}^{\prime \prime} \hat{\mathcal{L}}^{\prime \prime} \hat{\mathcal{L}}^{\prime} W\left(l_{2}^{\prime} l_{1}^{\prime} k \mathcal{L}^{\prime \prime} ; \mathcal{L}^{\prime} l_{2}\right) .
\end{aligned}
$$

The potentials between the valence particles and the target are more complicated as they depend on three vectors:

$$
V_{\mathrm{iT}}(|\vec{R}+\vec{\rho}+\vec{r}|)=\sum_{k_{r}, k_{\rho}, k_{R}} V_{k_{r} k_{\rho} k_{R}}^{\mathrm{iT}}(R, \rho, r)\left[\left[Y_{k_{r}}(\hat{r}) \otimes Y_{k_{\rho}}(\hat{\rho})\right] \otimes Y_{k_{R}}(\hat{R})\right]_{00}
$$

In the Appendix we outline two methods of evaluating the potential multipole components $V_{k_{r} k_{\rho} k_{R}}^{\mathrm{iT}}(R, \rho, r)$. The results presented use the Gaussian expansion method. In either case, the potential matrix elements are

$$
\begin{gathered}
\left\langle\alpha^{\prime} ; J\left|V_{\mathrm{iT}}(\vec{R}, \vec{\rho}, \vec{r})\right| \alpha^{\prime \prime}: J\right\rangle=\sum_{k_{r}, k_{\rho}, k_{R}} \frac{V_{k_{r}, k_{\rho}, k_{R}}^{\mathrm{iT}}(r, \rho, R)}{(4 \pi)^{\frac{3}{2}}} \\
W\left(\mathcal{L}^{\prime \prime} L^{\prime \prime} \mathcal{L}^{\prime} L^{\prime} ; J k_{R}\right)\left\langle L^{\prime \prime} 0 k_{R} 0 \mid L^{\prime} 0\right\rangle\left\langle\left\langle l_{1}^{\prime \prime} 0 k_{r} 0 \mid l_{1}^{\prime} 0\right\rangle\left\langle l_{2}^{\prime \prime} 0 k_{\rho} 0 \mid l_{2}^{\prime} 0\right\rangle\right. \\
(-)^{J+\mathcal{L}^{\prime}-L^{\prime \prime}-k_{R}} \hat{k}_{r} \hat{k}_{\rho} \hat{k}_{R}{\hat{L^{\prime \prime}}}^{\prime \prime} \hat{l}_{1}^{\prime \prime} \hat{l}_{2}^{\prime \prime} \hat{\mathcal{L}}^{\prime \prime} \hat{\mathcal{L}}^{\prime}\left\{\begin{array}{ccc}
l_{1}^{\prime \prime} & k_{r} & l_{1}^{\prime} \\
l_{2}^{\prime \prime} & k_{\rho} & l_{2}^{\prime} \\
\mathcal{L}^{\prime \prime} & k_{R} & \mathcal{L}^{\prime}
\end{array}\right\} .
\end{gathered}
$$

\section{The projectile ground state wavefunction}

The projectile ground state is described by a three-body wavefunction [9], expressed in Jacobi coordinates $\vec{\rho}$ and $\vec{r}$ (see figure 1). The wavefunction is linear combination of components of good internal relative angular momenta $l_{1}$ and $l_{2}$. The intrinsic spins of clusters couple to a total projectile intrinsic spin $j$ with projection $\sigma$. As we include only central interactions with the target, $j$ and $\sigma$ are good quantum numbers. We can write the 
ground state as

$$
\Phi_{\mathrm{o}} \equiv \Phi_{\mathrm{o}}^{I M_{I}}=\sum_{l_{1} l_{2} j} \Phi_{I, M_{I}}^{l_{1}, l_{2}, j}(\vec{\rho}, \vec{r})
$$

where the individual components are

$$
\begin{gathered}
\Phi_{I, M_{I}}^{l_{1}, l_{2}, j}(\vec{\rho}, \vec{r})=\sum_{\lambda_{1} \lambda_{2} \mathcal{L} M_{\mathcal{L}} \sigma}\left\langle l_{1} \lambda_{1} l_{2} \lambda_{2} \mid \mathcal{L} M_{\mathcal{L}}\right\rangle\left\langle\mathcal{L} M_{\mathcal{L}} j \sigma \mid I M_{I}\right\rangle \\
Y_{l_{1} \lambda_{1}}(\hat{r}) Y_{l_{2} \lambda_{2}}(\hat{\rho}) \chi_{j \sigma}(\xi) U_{I l_{1} l_{2}}^{j}(\rho, r) .
\end{gathered}
$$

We are now in a position to determine the $\mathcal{C}$ coefficients in the partial wave expansion of the four-body adiabatic wavefunction, (eq. 10). The asymptotic form of the wavefunction is obtained by substituting for the asymptotic form of $\mathcal{X}_{\alpha^{\prime}: \alpha}^{J}$ from eq. 12 into eq. 10. Using the delta function $\delta_{\alpha^{\prime} \alpha} \equiv \delta_{l_{1}^{\prime} l_{1}} \delta_{l_{2}^{\prime} l_{2}} \delta_{\mathcal{L}^{\prime} \mathcal{L}} \delta_{L^{\prime} L}$, then

$$
\begin{array}{r}
\Psi(\vec{R}, \vec{\rho}, \vec{r}) \stackrel{R \rightarrow \infty}{\longrightarrow} \sum_{l_{1} l_{2} \mathcal{L} L J M_{J}} \mathcal{C}_{\alpha}^{J M_{J}}(\rho, r) \frac{F_{L}(K R)}{K R} \sum_{\lambda_{1} \lambda_{2} M_{\mathcal{L}} \Lambda} Y_{l_{1} \lambda_{1}}(\hat{r}) Y_{l_{2} \lambda_{2}}(\hat{\rho}) Y_{L \Lambda}(\hat{R}) \\
\left\langle l_{1} \lambda_{1} l_{2} \lambda_{2} \mid \mathcal{L} M_{\mathcal{L}}\right\rangle\left\langle\mathcal{L} M_{\mathcal{L}} L \Lambda \mid J M_{J}\right\rangle+\text { (outgoing waves). }
\end{array}
$$

The wavefunction must have the asymptotic form (eq. 9) of a pure Coulomb distorted wave in the entrance channel with the projectile in the ground state, i.e.

$$
\Psi(\vec{R}, \vec{\rho}, \vec{r}) \stackrel{R \rightarrow \infty}{\longrightarrow}\left(\sum_{l_{1} l_{2} j} \Phi_{I, M_{I}}^{l_{1}, l_{2}, j}(\vec{\rho}, \vec{r})\right) \Psi_{\vec{K}_{\mathrm{o}}}^{\text {Coul }}(\vec{R})+\text { (outgoing waves) . }
$$

The correct form of the coefficients $\mathcal{C}$ is

$$
\begin{array}{r}
\mathcal{C}_{\alpha}^{J M_{J}}(\rho, r)=4 \pi \sum_{j \sigma M_{\mathcal{L}} \Lambda} i^{L} e^{i \sigma_{L}} Y_{L \Lambda}^{*}(\hat{K}) \chi_{j \sigma}(\xi) U_{I l_{1} l_{2}}^{j}(\rho, r) \\
\left\langle\mathcal{L} M_{\mathcal{L}} j \sigma \mid I M_{I}\right\rangle\left\langle\mathcal{L} M_{\mathcal{L}} L \Lambda \mid J M_{J}\right\rangle,
\end{array}
$$

as can be confirmed by substituting for eq. 23 in eq. 21

$$
\begin{aligned}
\Psi(\vec{R}, \vec{\rho}, \vec{r}) & \stackrel{R \rightarrow \infty}{\longrightarrow} \sum_{l_{1} l_{2} j} \sum_{\lambda_{1} \lambda_{2} \mathcal{L} M_{\mathcal{L}} \sigma}\left\langle l_{1} \lambda_{1} l_{2} \lambda_{2} \mid \mathcal{L} M_{\mathcal{L}}\right\rangle\left\langle\mathcal{L} M_{\mathcal{L}} j \sigma \mid I M_{I}\right\rangle \\
& \chi_{j \sigma}(\xi) U_{I l_{1} l_{2}}^{j}(\rho, r) Y_{l_{1} \lambda_{1}}(\hat{r}) Y_{l_{2} \lambda_{2}}(\hat{\rho}) \\
& 4 \pi \sum_{L \Lambda} i^{L} e^{i \sigma_{L}} Y_{L \Lambda}^{*}(\hat{K}) Y_{L \Lambda}(\hat{R}) \frac{F_{L}(K R)}{K R}+\text { (outgoing waves) } .
\end{aligned}
$$


The adiabatic wavefunction is therefore

$$
\begin{gathered}
\Psi(\vec{R}, \vec{\rho}, \vec{r})=4 \pi \sum_{X} i^{L} Y_{L \Lambda}^{*}(\hat{K}) \chi_{j \sigma}(\xi) U_{I l_{1} l_{2}}^{j}(\rho, r) e^{i \sigma_{L}}\left\langle\mathcal{L} M_{\mathcal{L}} L \Lambda \mid J M_{J}\right\rangle \\
\left\langle\mathcal{L} M_{\mathcal{L}} j \sigma \mid I M_{I}\right\rangle \frac{\mathcal{X}_{\left(l_{1}^{\prime} l_{2}^{\prime}\right) \mathcal{L}^{\prime} L^{\prime}:\left(l_{1} l_{2}\right) \mathcal{L} L}^{J}(R, \rho, r)}{K R}\left[\left[Y_{l_{1}^{\prime}}(\hat{r}) \otimes Y_{l_{2}^{\prime}}(\hat{\rho})\right]_{\mathcal{L}^{\prime}} \otimes Y_{L^{\prime}}(\hat{R})\right]_{J M_{J}}
\end{gathered}
$$

where the sum $X$ is over $\left\{l_{1} l_{2} j \sigma L L^{\prime} \Lambda \mathcal{L} \mathcal{L}^{\prime} M_{\mathcal{L}} J M_{J} l_{1}^{\prime} l_{2}^{\prime}\right\}$.

\section{E. Elastic Scattering amplitudes and observables}

To calculate the scattering amplitude for outgoing waves in channel $I^{\prime}, M_{I}^{\prime}$ we start by comparing the projection of final state wavefunction $\Phi_{I^{\prime} M_{I}^{\prime}}$ from the four-body scattering wavefunction, with the asymptotic boundary conditions:

$$
\left\langle\Phi_{I^{\prime} M_{I}^{\prime}} \mid \Psi(\vec{R}, \vec{\rho}, \vec{r})\right\rangle \stackrel{R \rightarrow \infty}{\longrightarrow} \delta_{I^{\prime} I} \delta_{M_{I}^{\prime} M_{I}} \Psi_{\vec{K}}^{\operatorname{Coul}}(\vec{R})+\frac{e^{i K R-\eta \log (2 K R)}}{R} f_{I^{\prime} M_{I}^{\prime}: I M_{I}}(\vec{K}, \vec{R})
$$

The scattering amplitude will be a linear combination of terms depending on the angular momentum components of the initial and final states

$$
f_{I^{\prime} M_{I}^{\prime}: I M_{I}}(\vec{K}, \vec{R})=\sum_{l_{1} l_{2} j l_{1}^{\prime} l_{2}^{\prime} j^{\prime}} f_{I^{\prime} M_{I}^{\prime} ; I M_{I}^{\prime}}^{l_{1}^{\prime} j^{\prime} l_{1} l_{2} j}(\vec{K}, \vec{R})
$$

where the individual components are given by:

$$
\begin{aligned}
& f_{I^{\prime} M_{I}^{\prime} ; I M_{I}}^{l_{1}^{\prime} l_{2}^{\prime} j^{\prime} l_{1} l_{2} j}(\hat{K}, \hat{R})=\frac{4 \pi}{K} \sum_{\mathcal{L} \mathcal{L}^{\prime} L L^{\prime} \Lambda \Lambda^{\prime} J M_{J} k}(2 J+1) \mathcal{M}_{\alpha^{\prime}: \alpha}^{J} \\
& i^{L-L^{\prime}} Y_{L \lambda}^{*}(\hat{K}) Y_{L^{\prime} \lambda^{\prime}}(\hat{R}) e^{i\left(\sigma_{L}+\sigma_{L}^{\prime}\right)} \hat{I} \hat{k}(-)^{\mathcal{L}^{\prime}-\mathcal{L}-J+k+j-I^{\prime}+\Lambda^{\prime}} \\
& \left\langle L \Lambda L^{\prime}-\Lambda^{\prime} \mid k q\right\rangle\left\langle I M_{I} k q \mid I^{\prime} M_{I}^{\prime}\right\rangle W\left(I \mathcal{L} I^{\prime} \mathcal{L}^{\prime} ; j k\right) W\left(L \mathcal{L} L^{\prime} \mathcal{L}^{\prime} ; J k\right) .
\end{aligned}
$$

The scattering matrix $\mathcal{M}_{\alpha^{\prime}: \alpha}^{J}$ is the matrix element of the internal coordinate dependent amplitudes $M_{\alpha^{\prime}: \alpha}^{J}(R, \rho, r)$, from eq. 12 , between the internal radial wavefunctions of the initial and final states:

$$
\mathcal{M}_{\alpha^{\prime}: \alpha}^{J}=\int \mathrm{d} r \int \mathrm{d} \rho U_{I^{\prime} l_{1}^{\prime} l_{2}^{\prime}}^{j *}(\rho, r) M_{\alpha^{\prime}: \alpha}^{J}(R, \rho, r) U_{I l_{1} l_{2}}^{j}(\rho, r) .
$$

For elastic scattering the radial wavefunctions $U_{I l_{1} l_{2}}^{j}$ are determined by the ground state wavefunction through eq. 20. The elastic cross section is given by

$$
\frac{\mathrm{d} \sigma_{\mathrm{el}}}{\mathrm{d} \Omega}(\theta, \phi)=\sum_{M_{I}^{\prime} M_{I}} \frac{1}{2 I+1}\left|\delta_{M_{I} M_{I}^{\prime}} f_{C}(\theta)+\sum_{l_{1} l_{2} j l_{1}^{\prime} l_{2}^{\prime} j^{\prime}} f_{I M_{I}^{\prime} ; I M_{I}^{\prime}}^{l_{1}^{\prime} l_{2}^{\prime} j^{\prime} l_{1} l_{2} j}(\theta, \phi)\right|^{2}
$$


where $f_{C}(\theta)$ is the pure Coulomb scattering amplitude, and the summation is over all the components of the ground state.

One of the advantages of our method is that, for elastic scattering, the internal dynamics of the projectile only enter through the ground state wavefunction. Excited state configurations are built in to the four-body wavefunction through the parameter dependence of the scattering wavefunction on $\vec{\rho}$ and $\vec{r}$, i.e. through the dynamics of the scattering of the individual clusters from the target.

\section{APPLICATION TO THE ELASTIC SCATTERING OF ${ }^{11}$ LI FROM ${ }^{12} \mathrm{C}$}

\section{A. Details of the calculation}

We now discuss the application of the model to elastic scattering of ${ }^{11} \mathrm{Li}+{ }^{12} \mathrm{C}$. The same projectile wavefunction and potentials are used as in the eikonal model calculations of [1] to allow comparison of the two models. The reasoning behind, and the sensitivity to the choice of wavefunction and potentials is described in [1]. Experimental quasi-elastic scattering data also exists for this system at $\mathrm{E}_{\mathrm{LAB}}=637 \mathrm{MeV}[8]$.

The projectile three-body wavefunction is from the Faddeev analysis of the ${ }^{11} \mathrm{Li}$ ground state by Thompson and Zhukov [9]. We use the 'P0' variant (labelled 'O7' in ref. [1]) which has two components: an s-wave $\left(l_{1}=l_{2}=0\right)$; and a p-wave $\left(l_{1}=l_{2}=1\right)$ component. As we neglect spin dependence in the target-cluster potentials, these two components will not couple and can be treated separately. Initially we concentrate on the s-wave component, which we renormalise to unity. The cross section for the full ground state wavefunction can be obtained by adding the amplitudes for s-wave and p-wave components with the correct normalisations.

The target-cluster potentials are also the same as used [1]. These have Woods-Saxon form factors with the parameters given in table 1 . 


\begin{tabular}{|l|l|l|l|l|}
\hline channel & form & depth $(\mathrm{MeV})$ & radius $(\mathrm{fm})$ & diff $(\mathrm{fm})$ \\
\hline 9Li+12C & Real WS & 120.0 & 0.70 & 0.90 \\
& Imag WS & 25.00 & 0.98 & 0.75 \\
\hline \multirow{2}{*}{$\mathrm{n}+12 \mathrm{C}$} & Real WS & 37.4 & 1.20 & 0.75 \\
& Imag WS & 10.0 & 1.30 & 0.60 \\
\hline
\end{tabular}

To calculate the valence neutron-target potential matrix elements we use the Gaussian expansion method (see Appendix). The Woods-Saxon potentials were approximated by a sum of five Gaussian terms, with radii in a geometric progression [12] (ranging from 1.8 to $2.65 \mathrm{fm})$. The strengths were obtained by least-squares fitting. The coupling potentials calculated using this expansion agree well with those calculated by direct numerical integration for $\rho$ and $r$ less than $9 \mathrm{fm}$ (see Appendix). In the multipole expansions we include terms up to $k_{\rho}, k_{r}=2$ in $\rho$ and $r$ and up to $k=4$ in $R$.

The Coulomb potential has been included only as a central term between the target and projectile centre of mass, and as a consequence this analysis ignores Coulomb breakup contributions. It has been shown previously that these have little effect on the elastic cross section for this reaction [1].

The coupled equations are solved using the Numerov algorithm (as used in FRESCO [13]), for 161 partial waves with step size (in $R$ ) $\mathrm{h}=0.02 \mathrm{fm}$ and matching radius $17 \mathrm{fm}$. The scattering amplitudes were obtained by matching to Coulomb functions at the matching radius. The calculations were repeated for a grid of values of the projectile internal coordinates, $\rho$ an $r$.

The grid is chosen to optimise the discretisation of the integration over $\rho$ and $r$. The smallest 6 values correspond to the radii for Gauss-Legendre integration and any further points are at regular intervals. We present results for $6 \times 6,12 \times 12$ and $18 \times 18$ point grids in $\rho$ and $r$ (where $6 \times 6$ refers to a 36 point grid composed of 6 points in the $\rho$ and $r$ coordinates). The $\rho$ and $r$ integrations are truncated beyond $9 \mathrm{fm}$. The eikonal calculations of ref. [1] used an integration grid of $30 \times 30$ points.

Whereas the four-body eikonal model [1] implicitly includes all breakup relative angular momenta, in our formulation we must specify explicitly which breakup angular momenta are 
included in the relative coordinates $\rho\left(l_{2}\right)$ and $r\left(l_{1}\right)$. As more angular momenta are included the number of coupled channels increases. We study the convergence as more angular momenta are included. For projectiles with two identical valence particles (e.g. $\left.{ }^{11} \mathrm{Li}\right)$, and assuming central potentials, the parity of the relative motion in $r$ must be conserved so $l_{1}$ can only change by an even number $\left(\Delta l_{1}\right.$ is even).

To illustrate the effect of breakup, we compare our results with a calculation with no breakup or excited state contributions. The 'no breakup' cross section is calculated using a two-body model with the potential calculated by averaging the cluster-target potential $V(\vec{R}, \vec{\rho}, \vec{r})$ over the internal coordinates, weighted by the ground state probability distribution:

$$
V^{\text {no b.u. }}(R)=\left\langle\Phi_{I, M_{I}}^{l_{1}, l_{2}, j}(\vec{\rho}, \vec{r})|V(\vec{R}, \vec{\rho}, \vec{r})| \Phi_{I, M_{I}}^{l_{1}, l_{2}, j}(\vec{\rho}, \vec{r})\right\rangle
$$

where $\Phi_{I, M_{I}}^{l_{1}, l_{2}, j}$ is a component of projectile ground state described in subsection 2.4 (renormalised to unity). A separate potential must be calculated for each component of the ground state.

\section{B. Results}

Initially we assume the projectile ground state is entirely s-wave. We consider the effects of including different breakup angular momenta, and of varying the number of grid points in the discretised integration over $\rho$ and $r$.

Figure 2 shows the four-body adiabatic model calculations for elastic scattering cross section of ${ }^{11} \mathrm{Li}+{ }^{12} \mathrm{C}$ at $637 \mathrm{MeV}$. All calculations use the same $6 \times 6$ point integration grid in $\rho$ and $r$, and differ only in the number of breakup angular momentum channels included. The 'no breakup' result (dotted) neglects all breakup contributions. The thin solid line, labelled $(0,0)$, includes coupling to s-wave breakup in the $\rho$ and $r$ coordinates. These couplings reduce the elastic cross section between $8^{\circ}$ and $18^{\circ}$ by about $10 \%$.

The remaining curves show the effects of including coupling to higher breakup angular momenta, where we introduce a shorthand notation $\left(l_{r}, l_{\rho}\right)$ for each channel. Including relative p-wave, $(0,1)$, and d-wave, $(0,2)$, breakup in the $\rho$ coordinate both reduce the cross 
section, and the combined effect is about a $40 \%$ reduction for angles greater than $8^{\circ}$. The effect of including relative $\mathrm{d}$-wave in the $r$ coordinate, $(2,0)$ is less significant. We have also calculated the effects of coupling to f-wave in $\rho,(0,3)$, and g-wave in $r,(4,0)$. These have no significant effect on the cross section and are not shown in the figure. It should be remembered that since we have only included coupling potential terms $l_{1}, l_{2} \leq 2$, channels with f-wave in $\rho$ and g-wave in $r$ can only be reached in two or more steps.

For the combination of $\mathrm{s}, \mathrm{p}, \mathrm{d}$ waves in $\rho$ and $\mathrm{s}, \mathrm{d}$ in $r$, (which we shall refer to as (spdd)) we have presented two results. The thick solid line shows the cross section when we include channels $(0,0),(0,1),(0,2),(2,0)$, which can all be reached in one step. The dot-dashed line shows the effect of adding the $(2,1)$ and $(2,2)$ channels, which can only be reached by two or more steps. The effects of these two-step channels is small and shall be neglected in the remaining calculations.

These calculations have included only $6 \times 6$ grid points in the $\rho$ and $r$ integration. We have repeated the calculations for the 'no breakup' and the spdd case with a $12 \times 12$ and a $18 \times 18$ point grid. Figure 3 shows the elastic cross section for these cases. Increasing the grid from $6 \times 6$ to $12 \times 12$ reduces the elastic cross section at angles greater than $8^{\circ}$. Extending the grid further from $12 \times 12$ to $18 \times 18$ has little effect on the 'no breakup' result but increases the cross section for the coupled (spdd) calculation at angles greater than $9^{\circ}$. However the cross sections show little sensitivity to our choice of integration grid.

\section{Comparison with eikonal model results}

In figure 4 we compare the results of the four-body adiabatic calculations (with the spdd couplings) and eikonal calculations for a range of projectile energies. The calculations assume the ${ }^{11} \mathrm{Li}$ ground state wavefunction is purely s-wave in both the $\rho$ and $r$ coordinates.

For energies greater than $20 \mathrm{MeV} / \mathrm{A}$ the adiabatic and eikonal model calculations are in reasonable agreement for forward angles but disagree at backward angles. At the higher energies, 637 and $1100 \mathrm{MeV}$, the adiabatic model gives a larger cross section than the eikonal model at angles beyond the first two minima. This may be because the adiabatic calculations do not include high enough breakup angular momenta. 
At the lower energies, 55, 110 and $220 \mathrm{MeV}$, the situation is reversed with the eikonal cross section higher than that of the adiabatic model at larger angles. A similar effect was observed in the comparison of two-body eikonal and exact quantum mechanical calculations in ref. [1]. This suggests that the deviation at low energies may be due to the eikonal approximation. Further work is required to clearly identify the validity of each approach as a function of energy and angle.

So far we have ignored the p-wave component of the projectile ground state. Figure 5 shows the adiabatic model predictions for the elastic cross section at $637 \mathrm{MeV}$ assuming the ground state is purely p-wave. The figure shows the effect of including channels with different breakup angular momenta. Several channels show a significant effect for the largest calculation attempted, including $(1,1),(1,0),(1,2),(1,3),(3,1)$. This calculation includes all channels that can be reached in one step with the potentials expanded to $l_{1}, l_{2} \leq 2$. Breakup has a smaller effect on the p-wave component of the ground state, compared with that on the s-wave component, with the maximum effect being a reduction of about $27 \%$ at about $13^{\circ}$ compared to the "no breakup' cross section.

In figure 6 we show the cross section using the full ground state wavefunction (with both s-wave and p-wave components). The figure also shows the four-body eikonal model calculation [1] and the experimental quasi-elastic cross section data from Kolata et al. [8]. There is a significant difference between the predictions of two models beyond $10^{\circ}$, where the adiabatic cross section is more than $10 \%$ larger.

\section{SUMMARY AND CONCLUSIONS}

A four-body quantum mechanical scattering model has been developed, which can include the effects of projectile breakup without making the semi-classical eikonal approximation. The elastic cross sections predictions of the model have been compared with four-body eikonal model predictions and with experimental data. The two methods give qualitatively similar results, particularly at forward angles, but deviate at the highest and lowest energies considered.

The disagreement at the higher energies may reflect non-convergence of the adiabatic calculations with respect to breakup angular momenta. While the eikonal model implicitly 
includes all angular momenta, it makes the additional straight-line trajectory approximation. The deviation at the lower energies may be due to the failure of the eikonal approximation, limiting the angular range. These questions will be addressed in a subsequent analysis in which we shall compare the exact adiabatic method developed here with calculations in which non-eikonal corrections are added systematically to the analysis of [1]. Such an analysis has proved very instructive for two-body projectiles [11].

As well as being an 'exact' quantum mechanical method, the adiabatic model has two additional advantages over the eikonal descriptions. It provides scattering wavefunctions, which may be used as input to reaction calculations. It also allows the individual contributions of different breakup angular momenta between the clusters in the projectile to be investigated. The results presented here indicate that for the s-wave component of the ${ }^{11} \mathrm{Li}$ wavefunction, s-, p- and d- wave breakup contributions are important. The coupling to breakup in the $\rho$ coordinate, between the ${ }^{9} \mathrm{Li}$ core and the centre of mass of the valence particles, is the most important. Breakup from the p-wave component of the ground state has a smaller effect.

There are several limitations to the calculations presented. We have used a fairly coarse integration grid in the $\rho$ and $r$ coordinates and restricted the range of this integration to $\rho, r<9 \mathrm{fm}$. These restrictions are not intrinsic but are to reduce computing time. Similarly we have limited the expansion of the coupling potentials to quadrupole contributions in the relative coordinates, $\rho$ and $r$.

We have demonstrated the feasibility of adiabatic four-body coupled channels calculations for exotic nuclear scattering. Further work is required to assess the convergence properties and the energy range for which the calculations are applicable. These limits will depend on the mass ratios of the clusters within the projectile, so studies with other three-body projectiles (e.g. ${ }^{6} \mathrm{Li},{ }^{6} \mathrm{He}$ and the triton) are planned.

\section{ACKNOWLEDGEMENTS}

This research is supported by the Engineering and Physical Sciences Research Council (UK) through grant GR/J95867. We would like to thank Dr. Ian Thompson for providing tabulated three-body wavefunction for ${ }^{11} \mathrm{Li}$. 


\section{APPENDIX A: APPENDIX: EVALUATION OF POTENTIAL MULTIPOLES}

We shall describe two methods for evaluating the four-body potential components $V_{k_{r} k_{\rho} k_{R}}^{i T}(R, \rho, r)$. The first method uses a Gaussian expansion that has proved efficient in other few-body studies [12]. The second method uses two numerical angular integrations, which has proved to be equally efficient.

\section{Gaussian expansion technique}

If we express the potential as a sum of Gaussians, then the coupling potentials can be evaluated analytically. To illustrate this we will consider a single Gaussian $V(x)=$ $V_{0} \exp \left(-b x^{2}\right)$ where $x=|\vec{R}+\vec{\rho}+\vec{r}|$. We can rewrite this potential as

$$
\begin{aligned}
& V(|\vec{R}+\vec{\rho}+\vec{r}|)=V_{0} \exp \left(-b\left[R^{2}+\rho^{2}+r^{2}\right]\right) \\
& \quad \times \exp (-2 b \vec{R} \cdot \vec{\rho}) \exp (-2 b \vec{\rho} \cdot \vec{r}) \exp (-2 b \vec{r} \cdot \vec{R}) .
\end{aligned}
$$

The exponentials of a scalar product can be expanded in partial waves such that

$$
V(|\vec{R}+\vec{\rho}+\vec{r}|)=\sum_{k_{r}, k_{\rho}, k_{R}} V_{k_{r} k_{\rho} k_{R}}(R, \rho, r)\left[\left[Y_{k_{r}}(\hat{r}) \otimes Y_{k_{\rho}}(\hat{\rho})\right] \otimes Y_{k_{R}}(\hat{R})\right]_{00},
$$

with coefficients given by

$$
\begin{aligned}
& V_{k_{r} k_{\rho} k_{R}}(R, \rho, r)=(4 \pi)^{\frac{3}{2}} V_{0} \exp \left(-b\left[R^{2}+\rho^{2}+r^{2}\right]\right) \sum_{k_{1}, k_{2}, k_{3}} i^{k_{1}+k_{2}+k_{3}} \\
& (-)^{k_{\rho}+k_{r}-k_{2}}\left\langle k_{1} 0 k_{3} 0 \mid k_{R} 0\right\rangle\left\langle k_{2} 0 k_{1} 0 \mid k_{\rho} 0\right\rangle\left\langle k_{3} 0 k_{2} 0 \mid k_{r} 0\right\rangle \\
& \left(2 k_{1}+1\right)\left(2 k_{2}+1\right)\left(2 k_{3}+1\right) W\left(k_{\rho} k_{1} k_{r} k_{3} ; k_{2} k_{R}\right) \\
& j_{k_{1}}(i 2 b R \rho) j_{k_{2}}(i 2 b \rho r) j_{k_{3}}(i 2 b r R)
\end{aligned}
$$

where $j_{k}(i x)$ is the spherical Bessel function for an imaginary argument.

\section{Numerical integration method}

An alternative method of obtaining the potential is to factor out the dependence on the angle between $\vec{\rho}$ and $\vec{r}$. The remaining term is dependent on the angle between $\vec{R}$ and vector $\operatorname{sum}(\vec{\rho}+\vec{r})$,

$$
V(|\vec{R}+\vec{\rho}+\vec{r}|)=\sum_{k_{R}}|\vec{\rho}+\vec{r}|^{k_{R}} P_{k_{R}}(\vec{R} \cdot(\vec{\rho}+\vec{r})) \sum_{k_{2}} P_{k_{2}}(\vec{r} \cdot \vec{\rho}) V_{k_{R}, k_{2}}(R, \rho, r) .
$$


The partial wave expansion of the potential can be expressed as

$$
V(|\vec{R}+\vec{\rho}+\vec{r}|)=\sum_{k_{r}, k_{\rho}, k_{R}} V_{k_{r} k_{\rho} k_{R}}(R, \rho, r)\left[\left[Y_{k_{r}}(\hat{r}) \otimes Y_{k_{\rho}}(\hat{\rho})\right] \otimes Y_{k_{R}}(\hat{R})\right]_{00}
$$

with the coefficients given by

$$
\begin{gathered}
V_{k_{r} k_{\rho} k_{R}}(R, \rho, r)=\sum_{k_{2} k_{3}}\left[\frac{2 k_{R} !}{2 k_{3} ! 2\left(k_{R}-k_{3}\right) !}\right]^{1 / 2}(4 \pi)^{3 / 2} r^{k_{R}-k_{3}} \rho^{k_{3}} \\
\left\langle k_{2} 0 k_{3} 0 \mid k_{\rho} 0\right\rangle\left\langle k_{2} 0\left(k_{R}-k_{3}\right) 0 \mid k_{r} 0\right\rangle(-1)^{k_{R}-k_{3}+k_{\rho}} \\
W\left(k_{\rho} k_{3} k_{r}\left(k_{R}-k_{3}\right) ; k_{2} k_{R}\right) V_{k_{R} k_{2}}(r, \rho, R),
\end{gathered}
$$

where the $V_{k_{R} k_{2}}(r, \rho, R)$ terms are determined by an integral over two angles

$$
\begin{gathered}
V_{k_{R} k_{2}}(r, \rho, R)=\frac{\hat{k}_{R}^{2} \hat{k}_{2}^{2}}{2} \int_{-1}^{1} \mathrm{~d} x_{1} \int_{-1}^{1} \mathrm{~d} x_{2} \frac{P_{k_{R}}\left(x_{1}\right) P_{k_{2}}\left(x_{2}\right)}{\left[r^{2}+\rho^{2}+2 r \rho x_{2}\right]^{k_{R} / 2}} \\
V\left(\left[r^{2}+\rho^{2}+2 r \rho x_{2}+R^{2}+2\left[r^{2}+\rho^{2}+2 r \rho x_{2}\right]^{1 / 2} R x_{1}\right]^{1 / 2}\right) .
\end{gathered}
$$

\section{Comparison of the two methods}

We have compared the potentials produced by both techniques for the neutron-target potential in ${ }^{11} \mathrm{Li}+{ }^{12} \mathrm{C}$. The agreement is excellent at most radii. The Gaussian expansion technique becomes inaccurate at large radii $r>9 \mathrm{fm}$ and $\rho>9 \mathrm{fm}$ due to truncation and round off errors. The numerical integration method is stable at large radii but is unstable for very small radii $(R, \rho, r<0.1 \mathrm{fm})$. Cross sections calculated with potentials obtained by the two different methods are in excellent agreement.

[1] J.S. Al-Khalili, Nucl. Phys. A581 (1995) 315

J.S. Al-Khalili, I.J. Thompson and J.A. Tostevin, Nucl. Phys. A 581 (1995) 331.

[2] J.S. Lilley, M.A. Nagarajan, D.W. Banes, B.R. Fulton and I.J. Thompson, Nucl. Phys. A 463 (1987) 710.

[3] R.C. Johnson and P.J.R. Soper, Phys. Rev. C 1 (1970) 976.

[4] H. Amakawa, S. Yamaji, A. Mori and K. Yazaki, Phys. Lett. B 82 (1979) 13.

[5] I.J. Thompson and M.A. Nagarajan, Phys. Lett. B 106 (1981) 163. 
[6] M.A. Nagarajan, I.J. Thompson and R.C. Johnson Nucl. Phys. A 385 (1982) 525.

[7] Y. Sakuragi, M. Yahiro and M. Kamimura, Prog. Theor. Phys. Suppl. 89 (1986) 136.

[8] J.J. Kolata, M. Zahar, R. Smith, K. Lamkin, M. Belbot, R. Tighe, B.M. Sherill, N.A. Orr, J.S. Winfield, J.A. Winger, S.J. Yennello, G.R. Satchler and A.H. Wuosmaa, Phys. Rev. Lett. 69 (1992) 2631.

[9] I.J. Thompson and M.V. Zhukov, Phys. Rev. C 49 (1994) 1904.

[10] E.J. Stephenson, A.D. Bacher, G.P.A. Berg, V.R. Cupps, C.C. Foster, N. Hodiwalla, P.C. Li, J. Lisantti, D.A. Low, D.W. Miller, C. Olmer, A.K. Opper, B.K. Park, R. Sawafta, S.W. Wissink, J.A. Tostevin, D.A. Coley, and R.C. Johnson, Phys.Rev. C 42, (1990) 2562.

J.A. Tostevin and B. Gönül, Genshikaku Kenkyu, 40 (1995) 95-104.

[11] J.S. Al-Khalili, J.A. Tostevin and J.M. Brooke, Phys. Rev. C, in press.

[12] M. Kamimura, Phys. Rev. A 38 (1988) 621.

[13] I.J. Thompson, Comp. Phys. Rep. 7 (1988) 167. 
Figure Captions

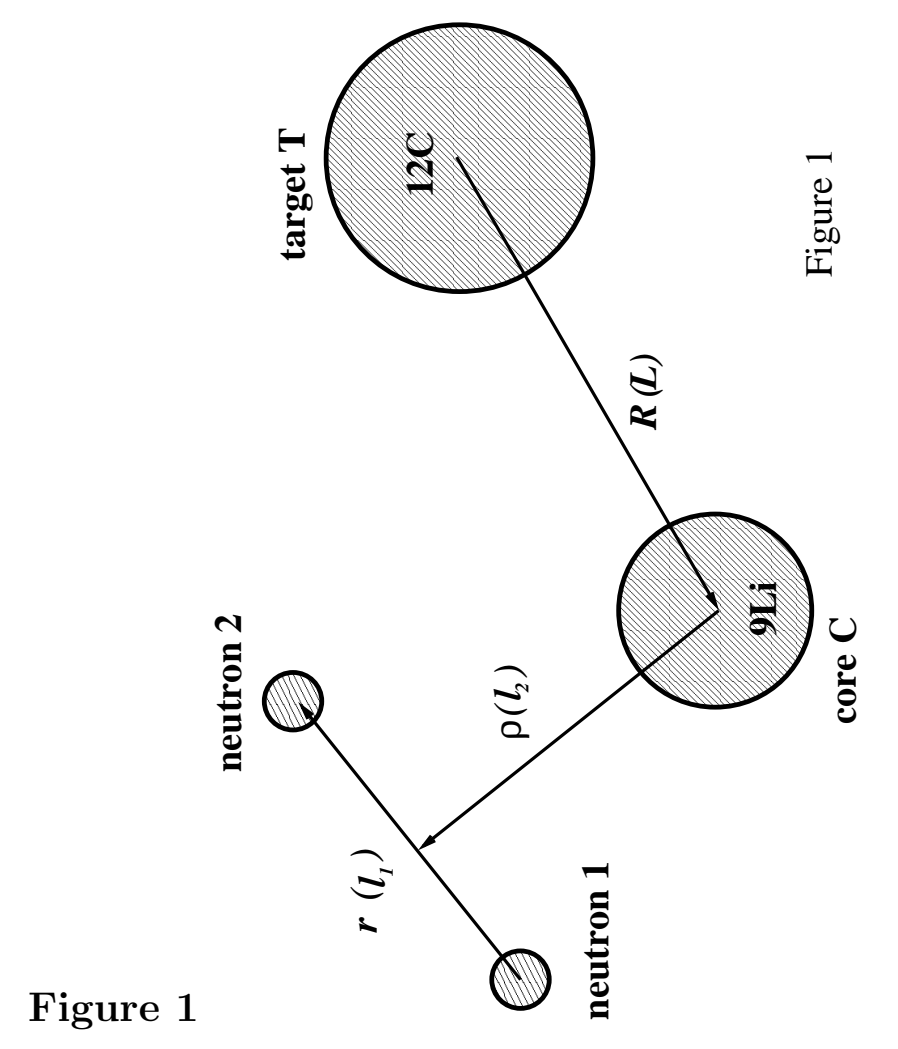

The coordinate system for a three cluster projectile scattering from and inert target (in this case ${ }^{11} \mathrm{Li}$ from ${ }^{12} \mathrm{C}$ ). The relative coordinates are $\vec{R}, \vec{\rho}, \vec{r}$ with corresponding relative angular momenta $L, l_{2}, l_{1}$. 
Figure 2

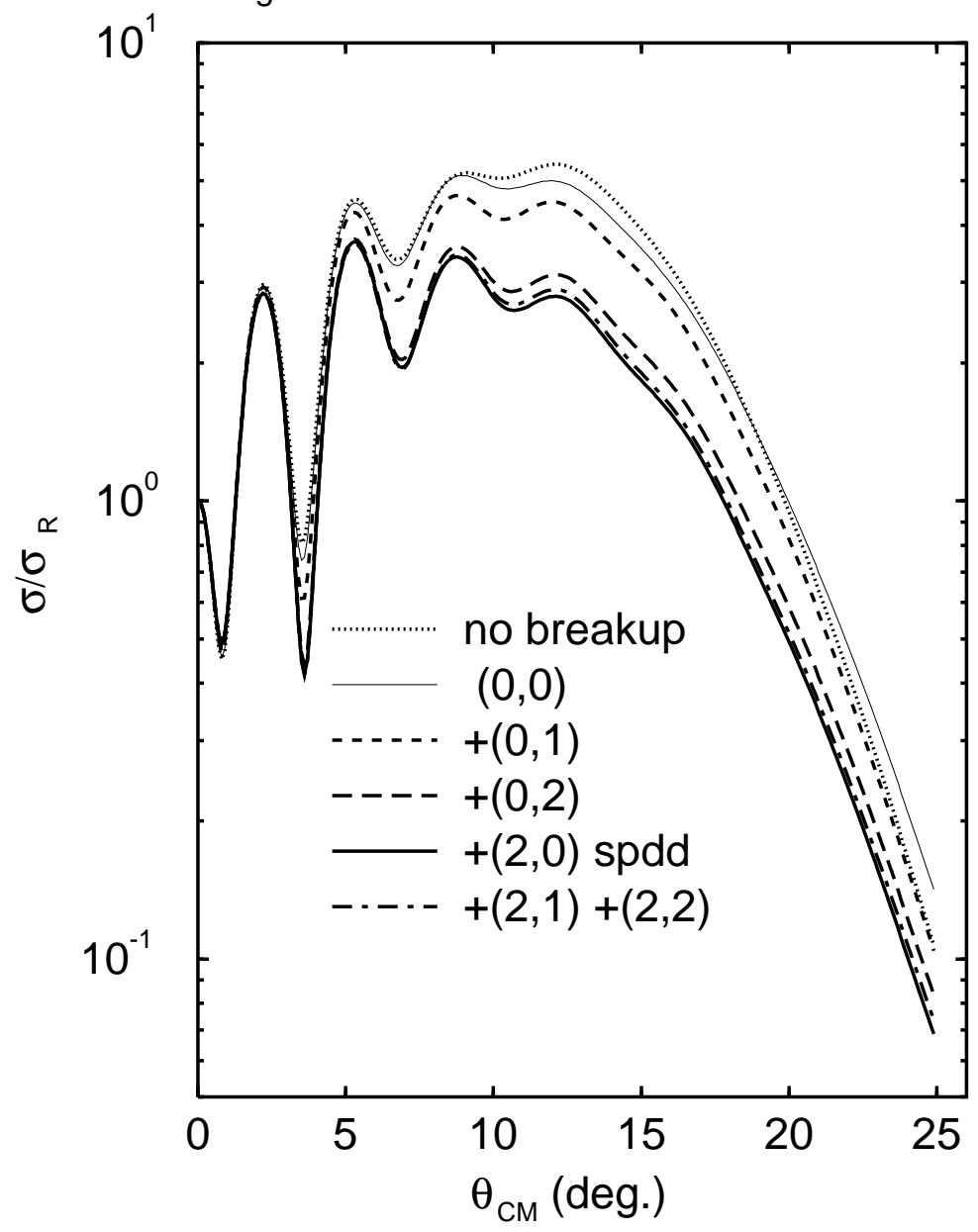

The elastic scattering cross section for ${ }^{11} \mathrm{Li}+{ }^{12} \mathrm{C}$ at $\mathrm{E}_{\mathrm{LAB}}=637 \mathrm{MeV}$ assuming the projectile ground state is purely s-wave. The 'no breakup' result is compared with 4-body adiabatic calculations that include successively more breakup relative angular momenta. The notation used to describe the channels is $\left(l_{1}, l_{2}\right)$ where $l_{1}$ is the relative angular momentum in the $r$ coordinate and $l_{2}$ is in the $\rho$ coordinate (see Figure 1) The converged result is the (spdd) calculation that includes s,p,d wave breakup in the $\rho$ coordinate and $\mathrm{s}, \mathrm{d}$ in $r$. 
Figure 3

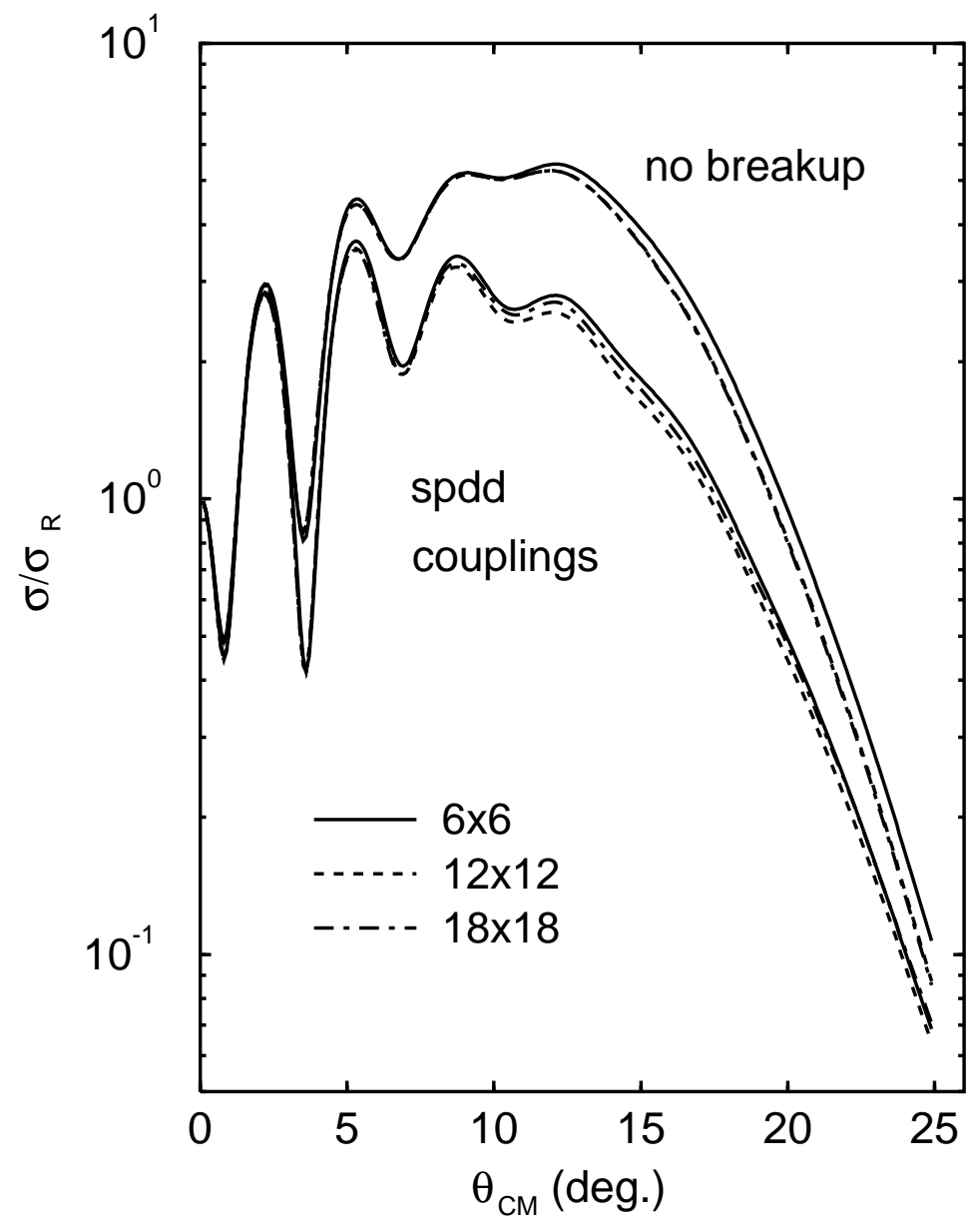

Figure 3

The 'no breakup' and (spdd) coupled cross sections for ${ }^{11} \mathrm{Li}+{ }^{12} \mathrm{C}$ at $637 \mathrm{MeV}$ are compared for three choices of the number of $\rho, r$ integration grid points. The 'no breakup' cross section appears to have converged for $12 \times 12$ points but the increase to $18 \times 18$ has an effect on the spdd cross section at large angles. The maximum value of $\rho$ and $r$ in all cases is $9 \mathrm{fm}$. 

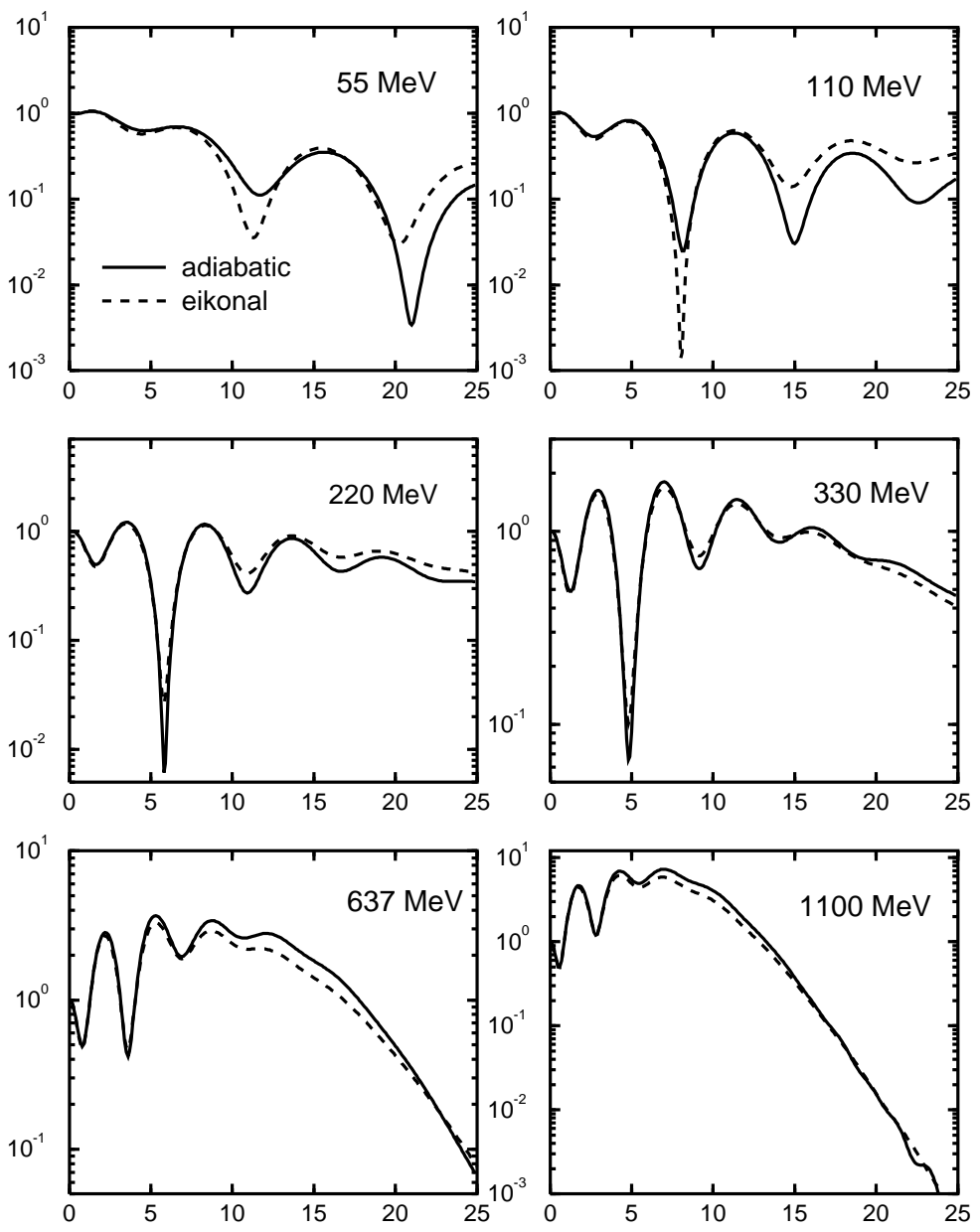

Figure 4

Figure 4

$\theta_{\mathrm{CM}}$ (deg.)

The elastic scattering of ${ }^{11} \mathrm{Li}+{ }^{12} \mathrm{C}$ for a range of projectile energies $\mathrm{E}_{\mathrm{LAB}}=55,110,220$, 330, 637 and $1100 \mathrm{MeV}$. For each energy we compare the four-body adiabatic calculation (using the spdd couplings) with four-body eikonal calculations. The projectile ground state is assumed to be purely s-wave. 
figure 5

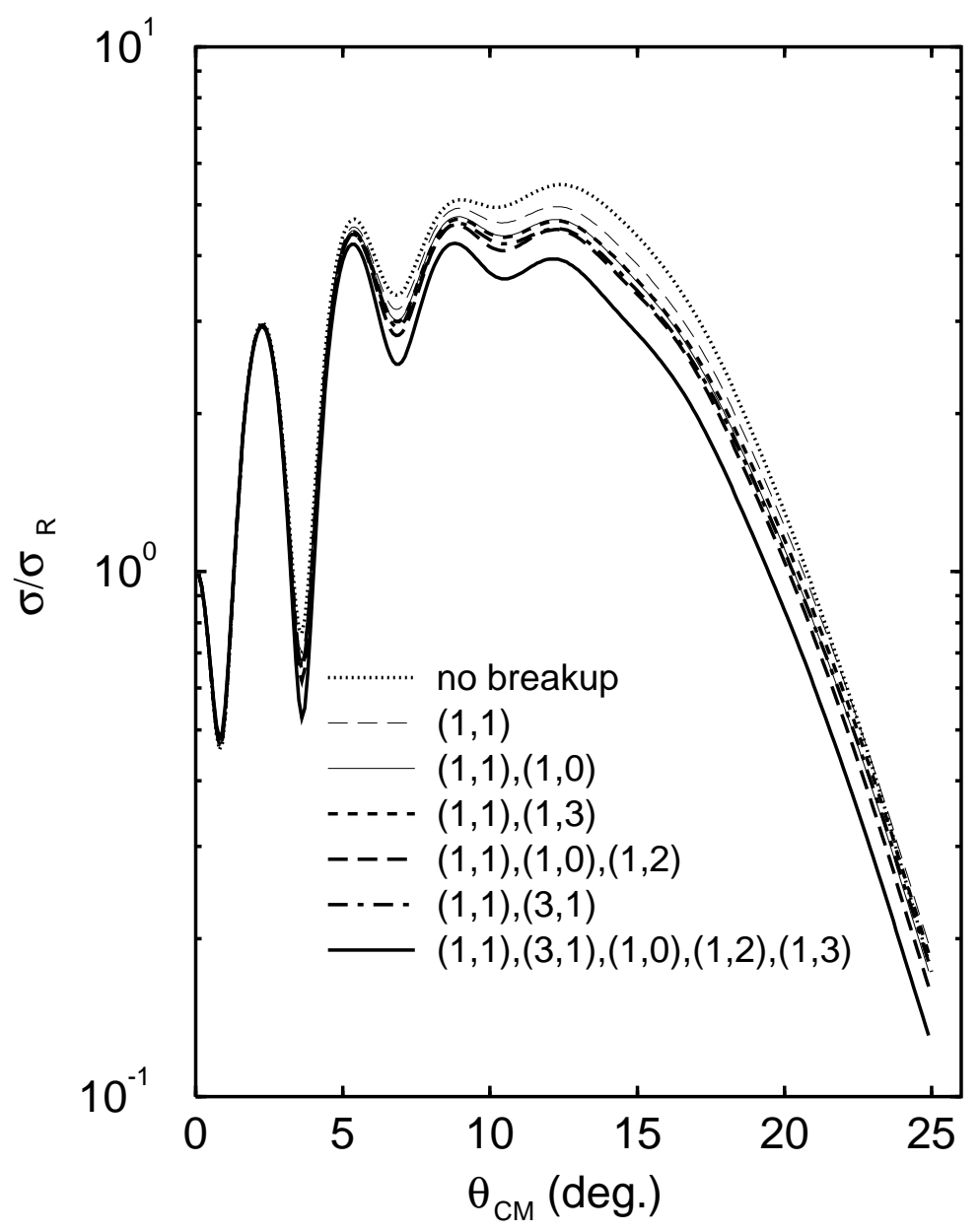

Figure 5

The elastic scattering of ${ }^{11} \mathrm{Li}+{ }^{12} \mathrm{C}$ at $637 \mathrm{MeV}$ assuming the projectile ground state is purely p-wave. We have use notation $\left(l_{1}, l_{2}\right)$ to indicate the channels included in the model. The dotted line is the 'no breakup' cross section and the thick solid line is the full calculation including coupling to s,p,d,g wave channels in $\rho$ and $\mathrm{p}, \mathrm{g}$ wave in $r$. 
Figure 6

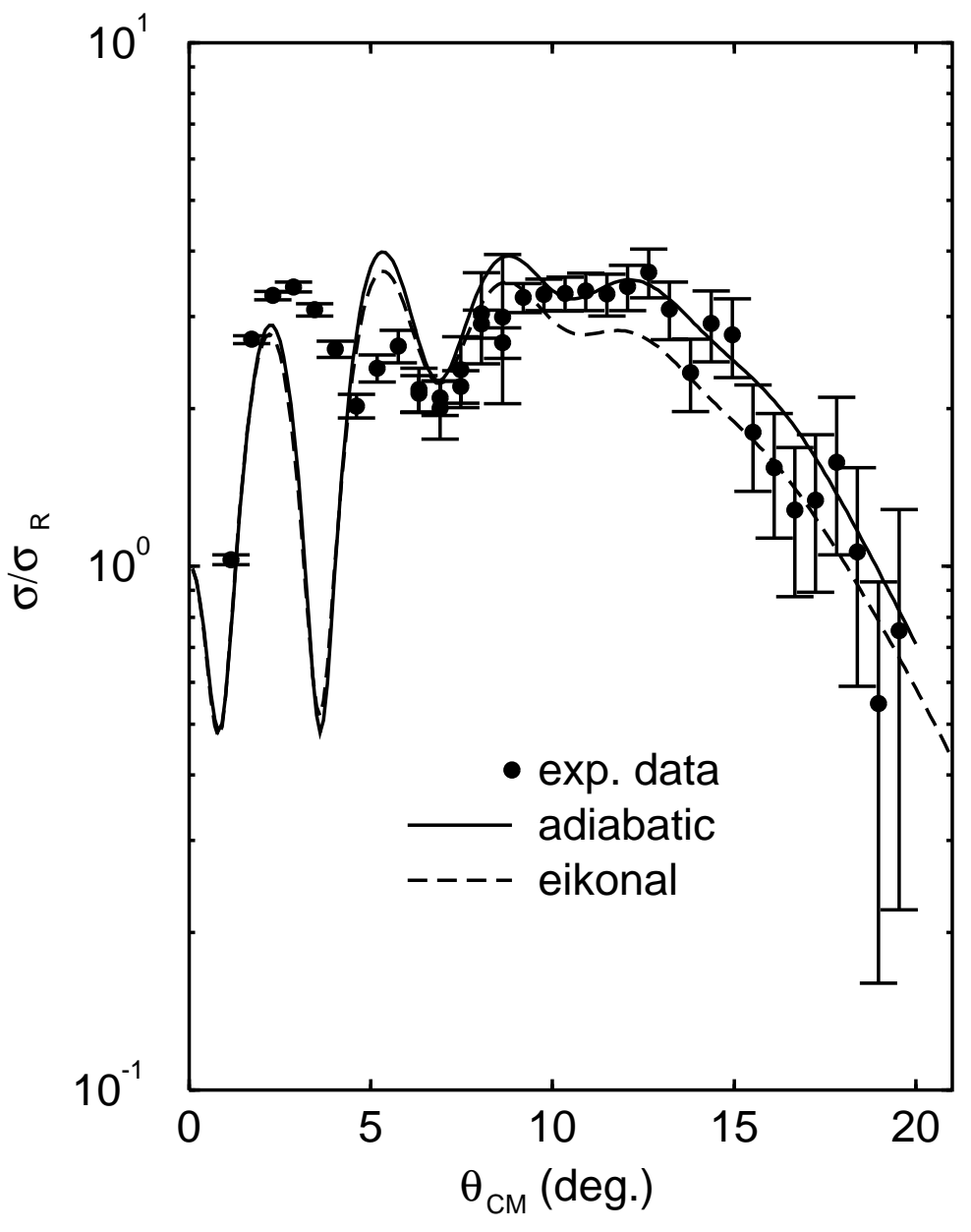

Figure 6

The elastic scattering of ${ }^{11} \mathrm{Li}+{ }^{12} \mathrm{C}$ at $637 \mathrm{MeV}$. The adiabatic and eikonal model results assume the projectile ground state has both s- and p-wave components (wavefunction 'P0' from ref. [9]). The experimental quasi-elastic cross section data are from Kolata et al. [8]. 